\title{
Abnormal Expression and Clinical Significance of Surface Receptors on Natural Killer Cells in the Peripheral Blood of Patients With Non-Small Cell Lung Cancer
}

pengcheng liu

Anhui Medical University

Xiaowen Feng

Anhui Medical University

Xiaomin Zhao

Anhui Medical University

Jing Ye

Anhui Medical University

Fenglian He

Anhui Medical University

Hui Liu

Anhui Medical University

Renming Li

Anhui Medical University

Xuefu Wang

Anhui Medical University

Dahai Zhao ( $\nabla$ zhaodahai@ahmu.edu.cn )

Anhui Medical University https://orcid.org/0000-0003-1500-5608

\section{Research Article}

Keywords: NK cell, NSCLC, immunity, prognosis

Posted Date: January 25th, 2022

DOI: https://doi.org/10.21203/rs.3.rs-1255893/v1

License: (1) This work is licensed under a Creative Commons Attribution 4.0 International License.

Read Full License 


\section{Abstract}

Background Natural killer (NK) cells typically function as frontline lymphocytes against cancer although little is known about their engagement in non-small cell lung cancer (NSCLC). This study compared the performance and activity of NK cells and their subsets in the peripheral blood of NSCLC sufferers and healthy participants.

Methods In total, 67 healthy controls (40 men; $59.7 \%$ ) and 56 patients with NSCLC (35 men; $62.5 \%$ ) were included (mean age, 66.6 years). Flow cytometry identified NK cells and their subpopulations in external blood, and the total number, proportion, activity, surface activating and inhibitory receptor expression levels were determined.

Results NK cell surface receptors CD107a, IFN- - , and TNF-a activity were markedly reduced in lung cancer patients compared to healthy controls. The number and ratio of NK cells within the lymphocyte population were decreased in patients. The concentration of the inhibitory receptors TIGIT, TIM-3, CD96, PD-1, and Siglec-7 were increased in patients, whereas the expression level of the activating receptor NKP30 was decreased. Moreover, the expression levels of IFN- $y$, TIGIT, CD96, PD-1, and TIM-3 were correlated with the clinical phase of NSCLC.

Conclusions NSCLC patients have decreased number and function of external blood NK cells, and the proportion of most of the inhibitory receptors assessed on NK cells in this study increased as the disease progressed. These findings suggest that surface receptors from NK cells are likely to be involved in the evolution of NSCLC.

\section{Introduction}

According to the global cancer burden report released by the International Agency for Research on Cancer [1], there were 18.1 million new patients with cancer worldwide in 2018, of which lung cancer ranked first with $11.6 \%$ of all cancers. As the pathologic type with the highest percentage among lung cancers, nonsmall cell lung cancer (NSCLC) often reaches phase IIIB-IV when diagnosed and is beyond the opportunity for aggressive treatment $[2,3]$. The treatment of advanced NSCLC is difficult, with low effective rates and limited benefits to patients. The survival rate of five years is approximately $16 \%[4]$. However, the use of precision medicine in cancer treatment is developing rapidly and showing promise [5]. Among those treatments, unlike traditional radiotherapy, chemotherapy, and targeted therapy, immunotherapy acts directly on the autoimmune system to restore the activity of immune cells, such as T cells and natural killer (NK) cells, by restraining immune checkpoint proteins from binding to their partners. The advantages of immunotherapy include a broad antitumor spectrum, long-lasting curative effect, minor adverse effects, and apparent synergistic effects with combined radiotherapy and chemotherapy, which can significantly extend the survival time of patients $[6,7]$. To date, most immunotherapy for lung cancer has focused on T cells, with few basic and clinical studies assessing NK cells. 
NK cells are distributed in the internal organs mainly in the lungs, followed by the liver [8]. These cells account for $5-20 \%$ of the circulating lymphocyte pool in the peripheral blood of normal grownups[9]. NK cells kill target cells directly without sensitization. Their activity depends on the stability of activating and inhibitory signals. NK cell-activating receptors include NKG2D, NKp30, NKp44, and NKp46; moreover, NK cell inhibitory receptors include CD96, NKG2A, and TIM-3, together with the T cell immunoglobulin and ITIM domain (TIGIT). In addition, NK cells generate cytokines and chemokines to regulate the immune response. This functional characteristic of NK cells suggests that the receptor feature of NK cells may be of great importance in the immunotherapy of lung cancer $[6,10]$. However, studies investigating this function have shortcomings, such as few studies have involved human biological samples and one-sided detection indicators [11].

Therefore, to assess evidence for the antitumor application of NK cells in NSCLC, the present study used flow cytometry to analyze the number, proportion, and expression levels of inhibitory and reactors activated from the surface of peripheral blood NK cells and their subpopulations in patients with NSCLC and healthy control participants.

\section{Methods}

\section{Antibodies}

The mentioned antibodies were used in this study: fluorescein isothiocyanate (FITC)-conjugated antihuman CD3 (OKT3), allophycocyanin (APC)-conjugated anti-human CD56 (5.1H11), Alexa Fluor ${ }^{\circledR} 700$ conjugated anti-human CD16 (3G8), PerCP/Cyanine5.5-conjugated anti-human TIGIT (TIGIT; A15153G), phycoerythrin (PE)-conjugated anti-human CD328 (Siglec-7; 6-434), allophycocyanin (APC)-conjugated anti-human Siglec-9 (K8), APC/Cyanine7-conjugated anti-human CD366 (Tim-3; F38-2E2), FITCconjugated anti-human CD279 (PD-1; A17188B), PE/Cyanine7-conjugated anti-human CD336 (NKP44; P44-8), PerCP/Cyanine5.5-conjugated anti-human CD314 (NkG2D; 1D11), PerCP/Cyanine5.5-conjugated anti-human CD335 (NKP46; 9E2), PE/Cyanine7-conjugated anti-human CD96 (NK92.39), PE-conjugated anti-human tumor necrosis factor (TNF)-a (MAb11), PerCP-conjugated anti-human CD107a (lysosomalassociated membrane protein 1 [LAMP-1]; H4A3), PE/Cyanine7-conjugated anti-human interferon (IFN)- $\mathrm{Y}$ (B27), PerCP/Cyanine5.5-conjugated anti-human CD337 (NKP30; P30-15). These antibodies and isotypes were obtained from BioLegend (San Diego, CA, USA).

\section{Human samples}

Participants included initially treated patients with NSCLC hospitalized in the Department of Respiratory Medicine of the Second Affiliated Hospital of Anhui Medical University from August to September 2021 who were consistent with the following criteria: (1) Histopathologic evidence supporting the diagnosis of NSCLC; (2) No previous cancer therapies, such as surgery, radiotherapy, chemotherapy, targeted treatments, and immunotherapy; (3) No missing clinical data; and (4) Patient and family members provided informed consent. The exclusion criteria included receiving a diagnosis of acquired immunodeficiency syndrome; a blood system disease, such as leukemia, lymphoma, or megaloblastic 
anemia; autoimmune disease, such as inflammatory bowel disease, vasculitis, or rheumatoid arthritis; or (4) other types of tumors. Healthy volunteers were selected during the same timeframe from the check-up center. The Ethics Committee of Anhui Medical University approved this study (YX2021-064[F1]). All parties provided a written informed consent form.

\section{Isolation of peripheral blood mononuclear cells (PBMCs)}

After fasting for a minimum of 12 hours, venous blood $(5 \mathrm{ml})$ was gathered from participants. Tubes containing EDTA anticoagulants were used to collect the samples, which were submitted for testing within 4 hours. PBMCs were identified from systemic blood with a Ficoll-Paque density gradient centrifugation method. Saline was used to resuspend the separated PBMCs.

\section{Flow cytometry}

PBMC suspensions were added to sample tubes containing the fluorescently labeled antibody. A Beckman Coulter flow cytometry system was used to detect NK cell phenotypic characteristics and the expression of CD16, CD56, IFN- - , TNF-a, CD107a, TIGIT, PD-1, TIM-3, Siglec-7, Siglec-9, CD96, NKG2D, NKp30, NKp44, and NKp46 in NK cells. FlowJo software was used to analyze the resulting data.

\section{Statistical analysis}

Data collected are displayed as mean \pm standard error and were analyzed using SPSS, version 22.0, software. We used independent-sample $t$-tests for metric data comparisons and chi-square tests for grade comparisons. Metric data were subjected to a normality test and a homogeneity of variance test. Values of $P<0.05$ indicated a statistical difference between the data, ${ }^{*} P<0.05 ; \star \star P<0.01 ;{ }^{*} \star * P<0.001$; ns, not significant.

\section{Results}

\section{Patient characteristics}

The study included 123 participants: 67 healthy volunteers and 56 patients with NSCLC (mean age, 66.6 years). Both chi-square and $t$-tests showed no statistically significant discrepancy in age or sex distribution between the control and the NSCLC groups. Participants in the control group had significantly lower tobacco smoking rates than those in the NSCLC group. Among the patients with NSCLC, 29 were diagnosed with early lung cancer (stage I or II), and 27 were diagnosed with advanced lung cancer (stage III or IV); 27 patients were identified with lung adenocarcinoma, and 29 patients with squamous cell carcinoma of the lung were diagnosed (Table 1). 
Table 1

Clinical and demographic characteristics of the study populations

\begin{tabular}{|lll|}
\hline Characteristic & $\begin{array}{l}\text { No. }(\%) \text { of healthy participants } \\
(\mathbf{n = 6 7 )}\end{array}$ & $\begin{array}{l}\text { No. }(\%) \text { of patients with NSCLC } \\
(\mathbf{n}=56)\end{array}$ \\
\hline Gender & $27(40.3)$ & $21(37.5)$ \\
\hline Female & $35(62.5)$ \\
\hline Male & $40(59.7)$ & \\
\hline Age & $29(43.3)$ & $23(41.1)$ \\
\hline$\geq 65$ years & $38(56.7)$ & $33(59.0)$ \\
\hline Smoking status & & $29(51.8)$ \\
\hline Ever-smoker & $19(28.4)$ & $27(48.2)$ \\
\hline Never-smoker & $48(71.6)$ & \\
\hline Stage & & $8(14.3)$ \\
\hline$\square$ & $21(37.5)$ \\
\hline$\square$ & & $13(23.2)$ \\
\hline$\square$ & & $14(25.0)$ \\
\hline$\square$ & & $29(16.1)$ \\
\hline ECOG performance & & \\
\hline status & & $27(84.0)$ \\
\hline Histologic subtype -4 & & $9(51.8)$ \\
\hline ADC & & \\
\hline SQcC & & \\
\hline
\end{tabular}

Abnormal number, frequency, and function of NK cells in the peripheral blood of patients with NSCLC

Flow cytometry analyses indicated that $\mathrm{CD}^{-} \mathrm{CD}^{+} 6^{+} \mathrm{NK}$ cells were initially recognized in the lymphoid gate and then further distinguished into $\mathrm{CD} 56^{\mathrm{dim}} \mathrm{CD} 16^{+}$and $\mathrm{CD} 56^{\text {bright }} \mathrm{CD} 16^{-}$NK subgroups (Figure $1 \mathrm{~A}$ ). The ratio(Figure 1B) and amount(Figure 1E) of total NK cells of NSCLC patients were remarkably reduced compared to the healthy volunteers. No remarkable differences between the healthy volunteers and NSCLC patients in the ratios of $\mathrm{CD} 56^{\mathrm{dim}} \mathrm{CD} 16^{+} \mathrm{NK}$ cell and $\mathrm{CD} 56^{\text {bright }} \mathrm{CD} 16^{-} \mathrm{NK}$ cell subgroup or for the 
cytometry of the CD56 ${ }^{\text {bright }}$ CD $16^{-}$NK cell subset in the NSCLC patients (Figure 1C, D, G). However, the count for the $\mathrm{CD} 56^{\mathrm{dim}} \mathrm{CD} 16^{+} \mathrm{NK}$ cell subgroup was remarkably decreased in the NSCLC group (Figure 1F). In addition, secretion of IFN-y, CD107a, and TNF-a from NK cells was decreased in patients with NSCLC (Figure 2). Thus, compared with healthy participants, patients with NSCLC showed a decrease in the ratio of NK cell subpopulations, cell numbers, and NK cell function.

\section{Activating and inhibitory receptors on the surface of NK cells in patients with NSCLC are abnormal and are associated with disease progression}

The equilibrium of activating and inhibiting signals regulates the physiological functions of NK cells. Thus, we compared the proportion of six inhibitory receptors and four activating receptors between NSCLC patients and healthy participants.

For the inhibitive receptors of NK cells, the ratio of $\mathrm{TIGIT}^{+}, \mathrm{TIM}^{+}{ }^{+}$, and $\mathrm{CD} 96^{+} \mathrm{NK}$ cells in the peripheral blood of NSCLC patients was significantly increased (Figure 3E, 3l; Figure 4E). Both the health and the NSCLC group had a poor percentage of PD-1+ NK cells but were remarkably higher in the NSCLC group (Figure $3 \mathrm{G}$ ). No significant difference was detected in the proportion of Siglec-7 $7^{+}$NK cells between the control and NSCLC groups, but the mean fluorescence intensity was remarkably reduced in the NSCLC group (Figure 3K, L). No significant group difference was found in the proportion or mean fluorescence intensity of Siglec-9 ${ }^{+}$NK cells (Figure 4C, D).

For the activating receptors, the proportion of NKP30 ${ }^{+}$NK cells in the NSCLC patients was noticeably lower than that in the healthy volunteers (Figure 5G). By contrast, the proportions and mean fluorescence

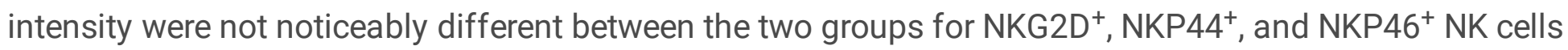
(Figure 5E, F, I, J, K, L).

To further explore the association between lung cancer and NK cell surface receptors, we divided the NSCLC group into different subgroups based on TNM staging and histopathological classification. We assessed the proportions of IFN- $\gamma^{+}, \mathrm{TIGIT}^{+}, \mathrm{TIM}^{+} 3^{+}, \mathrm{PD}-1^{+}$, and NKP30${ }^{+} \mathrm{NK}$ cells across these subgroups (Table 2). The study reveals that the proportion of NKP30+ NK cells is noticeably higher in the lung adenocarcinoma subgroup than in the squamous cell carcinoma subgroup, whereas the proportions of IFN- $\gamma^{+}, \mathrm{TIGIT}^{+}, \mathrm{TIM}^{-} 3^{+}$, and PD-1+ $\mathrm{NK}$ cells did not differ significantly between these two categories. The proportions of $\mathrm{TIGIT}^{+}, \mathrm{TIM}^{-} 3^{+}, \mathrm{PD}-1^{+}$, and $\mathrm{CD} 96^{+} \mathrm{NK}$ cells in the advanced lung cancer subgroup were greater than those in the early lung cancer subgroup, and the proportions of IFN- $\gamma^{+} \mathrm{NK}$ cells were higher than those in early days lung carcinoma group. No significant difference in NKP30 $0^{+} \mathrm{NK}$ cells was found between the two groups. 
Table 2

Association between NK cell surface receptors and disease progression, histologic types of nonsmall cell lung cancer

\begin{tabular}{|lllllll|}
\hline \multirow{2}{*}{ NK cell subset } & \multicolumn{5}{l}{ Cancer stage } & \multicolumn{3}{l|}{ Histologic type } \\
\cline { 2 - 7 } & Early (\%) & Late (\%) & P-value & ADC (\%) & SQCC (\%) & P-value \\
\hline${\mathrm{IFN}-\mathrm{y}^{+}}^{28.74 \pm 6.37}$ & $6.07 \pm 2.14$ & 0.010 & $14.74 \pm 6.94$ & $19.18 \pm 7.22$ & 0.685 \\
\hline $\mathrm{TIGIT}^{+}$ & $49.27 \pm 5.89$ & $66.41 \pm 4.50$ & 0.039 & $55.94 \pm 5.58$ & $57.57 \pm 6.58$ & 0.851 \\
\hline${\mathrm{TIM}-3^{+}}^{28.65 \pm 3.98}$ & $61.08 \pm 5.93$ & 0.004 & $41.97 \pm 7.21$ & $57.33 \pm 11.70$ & 0.268 \\
\hline $\mathrm{PD}-1^{+}$ & $1.58 \pm 0.27$ & $4.53 \pm 1.11$ & 0.009 & $2.79 \pm 0.78$ & $2.94 \pm 0.91$ & 0.899 \\
\hline $\mathrm{CD}^{+} 6^{+}$ & $64.70 \pm 1.98$ & $89.07 \pm 3.83$ & 0.005 & $74.95 \pm 7.43$ & $80.75 \pm 12.15$ & 0.687 \\
\hline $\mathrm{NKP}^{+} 0^{+}$ & $34.08 \pm 4.08$ & $28.03 \pm 4.06$ & 0.310 & $38.46 \pm 4.13$ & $24.38 \pm 3.09$ & 0.011 \\
\hline
\end{tabular}

These findings suggest that the expression of receptors on the NK cell surface is abnormal in the peripheral blood of NSCLC patients. In general, the expression of inhibitory receptors was high whereas activation receptor expression was low, and the expression of most cell surface inhibitory receptors was higher with disease progression, suggesting the activity decreased of NK cells was enhanced with disease progression. However, the expression of the various cell surface receptors did not appear to be associated with the histopathological type of lung neoplasms.

\section{Discussion}

Although treatments such as radiotherapy, chemotherapy, and targeted therapy have greatly extended the survival periods of lung neoplasms patients, long-term tumor control is still difficult to achieve, and a new treatment is urgently needed [12]. To this end, the present study sought to determine whether NK cell surface receptor expression and NK cell function are altered in the peripheral blood of NSCLC patients. We found that compared healthy participants, the number and proportion of NK cells in the peripheral blood of NSCLC patients was decreased, the anti-cancer activity of NK cells was decreased, and there was increased expression of NK cell surface inhibitory receptors TIGIT, CD96, TIM-3, and PD-1, In contrast, the activation receptor NKP30 expression was decreased. In addition, the expression of the inhibitory surface receptors was higher with a more advanced clinical stage of the tumor. This finding is consistent with those of recent studies $[13,14]$. Inhibitory receptors CD96, TIGIT, TIM-3, and PD-1 expression were similar in the patient groups suggests that future research investigating these receptors in NSCLC is warranted.

Studies have found a clear correlation between the body's immune function and the onset of tumors, with damage to the immune system substantially weakening its ability to monitor and kill tumors. Although increasingly more studies have found that the amount and function of immune cells in patients with 
tumors are typically abnormal, these studies have focused on $\mathrm{T}$ lymphocytes, with few studies assessing NK cells[15]. Moreover, previous research was carried out for patients with hematologic malignant neoplasms.

Recent research has shown that NK cells play a crucial role in selectively identifying and killing solid tumors, including NSCLC [16]. Under the guidance of chemokines, activated NK cells kill cancer cells through missing-self mechanisms, antibody-dependent cellular cytotoxicity, and activating caspase pathways to induce apoptosis. NK cells have considerable anti-cancer advantages, especially for advanced tumors [17]. When innate immunity and acquired immunity are in a non-responsive state, The killing of cancer cells by NK cells is still possible. Additional researchers have found that NK cell infiltration in lung cancer tissue is associated with a good prognosis for lung cancer patients[14].

Therefore, we believe that a decrease in the number of NK cells or abnormal function of these cells would be associated with an increase in the incidence and accelerate the progression of lung cancer. Most of the activating or inhibitory receptors of NK cells are phosphorylated through ITAM or ITIM motifs and recruit SHP-1, SHP-2, SHIP-1, and other phosphatases and bind them to activate downstream signal transduction [18-20].

Studies have found that the expression of CD96 is enhanced in patients with acute myeloid leukemia, chronic hepatitis B, and liver cirrhosis [21]. By competing with CD226 to bind CD155, CD96 directly inhibits the function of NK cells[22]. In addition, in a lipopolysaccharide-induced model of inflammation, mice that were $c d 96^{-/-}$showed stronger sensitivity and excellent anti-cancer characteristics when tested in subsequent tumor models. Despite these findings, most previous research on CD96 has been conducted in mice, with few studies assessing the role of CD96 in NSCLC in humans. However, the expression level of CD96 in mouse NK cells is significantly higher than that in humans, showing significant differences[23]. The TIGIT receptor is similar to CD96 in that both competitively bind CD155 and mediate cell adhesion and signal transmission [24]. In the human NK cell line YTS, after TIGIT binds to CD155, it inhibits the activation of TRAF6 and NF-KB through ITT-like motifs, which ultimately leads to a decrease in IFN- $\gamma$ production by NK cells[25]. Knockout of the TIGIT gene in NK cells partially restores the activity of NK cells and significantly reduces the ability of tumor-infiltrating killer T cells to secrete PD1. A common prognostic biomarker for tumors, the Tim-3 receptor is co-expressed with PD-1. In addition, interleukin 27 activates the expression of Tim-3 in T cells and induces transcription regulators that stimulate a module of co-inhibitory receptors that includes PD-1, TIM-3, TIGIT, and LAG3 [26]. Therefore, together with previous findings, our results suggest that reduced T cell and NK cell function is closely associated with the expression of CD96, TIGIT, TIM-3, and PD-1[27].

Immune checkpoint blockade is a promising treatment to reverse the functional reduction of immune cells. Antibodies against CTLA-4 and PD- 1 have been used in clinical practice and have achieved significant results. Our findings provide evidence supporting the combined blockade of multiple receptors as a solution to the treatment of NSCLC that is refractory to a single blocker, such as PD-1, which is the direction of future tumor treatment development [28]. 
Recent studies have found that the tumor microenvironment can damage the local function of NK cells [29]. Compared with NK cells in distal lung tissue or peripheral blood, NKp30, NKp80, and other receptors in tumor tissues are reduced in expression in NK cells, and the secretion of granzyme B is significantly decreased [30]. As a limitation of this study, we did not collect lung cancer tissues to evaluate NK cell surface receptors. In addition, the classification of inhibitory or activating NK cell surface receptors does not fully describe their functional characteristics. For example, the inhibitory receptor Siglec-7 shows an expression pattern similar to that of an activating receptor[31], suggesting that NK cell surface receptors may have other effects through unique signaling pathways. Follow-up research by our team will explore the signaling pathways of NK cell surface receptors associated with lung cancer progression.

In summary, NK cells demonstrate an essential role in the immune system against cancer cells. This study demonstrated that the percentage of peripheral blood NK cells in NSCLC patients was decreased, and the expression levels of various NK cell subgroups were abnormal. Inhibitory receptor expression on the surface of NK cells was significantly elevated, whereas the activation receptor expression was weak. The function of the NK cells was reduced and was associated with disease progression. These findings suggest that systematic analysis of NK cell surface receptor expression may aid in assessing the therapeutic effect of tumors and for guiding multi-drug combination immunotherapy so that patients may obtain greater treatment benefits. Further research investigating NK cells in lung cancer is warranted to supplement and improve lung cancer treatment strategies.

\section{Declarations}

\section{Acknowledgments}

This study was supported by the CAPTRA-Lung Research Funds (Grant No. CAPTRALung2020004), the Joint Fund between the Second Affiliated Hospital of Anhui Medical University and the Center of Medical Physics and Technology of Hefei Institute of Physical Sciences of Chinese Academy of Sciences (Grant No. LHJJ202002), Hefei Municipal Natural Science Foundation (Grant No. 2021037), the 2020 Schoollevel Scientific Research Fund of Anhui Medical University (Grant No. 2020xkji195) and the Research Fund of the Anhui Institute of Translational Medicine (2021zhyx-c67).

\section{Disclosure}

No authors report any conflict of interest.

\section{References}

1. Bray F, Ferlay J, Soerjomataram I, Siegel RL, Torre LA, Jemal A. Global cancer statistics 2018: GLOBOCAN estimates of incidence and mortality worldwide for 36 cancers in 185 countries. CA Cancer J Clin. 2018;68(6):394-424. https://doi.org/10.3322/caac.21492.

2. Rodríguez M, Ajona D, Seijo LM, Sanz J, Valencia K, Corral J, et al. Molecular biomarkers in early stage lung cancer. Transl Lung Cancer Res. 2021;10(2):1165-85. https://doi.org/10.21037/tlcr-20- 
750.

3. Tubin S, Khan MK, Gupta S, Jeremic B. Biology of NSCLC: Interplay between Cancer Cells, Radiation and Tumor Immune Microenvironment. Cancers (Basel).

2021;13(4):https://doi.org/10.3390/cancers13040775.

4. Lazzari C, Karachaliou N, Bulotta A, Viganó M, Mirabile A, Brioschi E, et al. Combination of immunotherapy with chemotherapy and radiotherapy in lung cancer: is this the beginning of the end for cancer? Ther Adv Med Oncol. 2018;10(1758835918762094

https://doi.org/10.1177/1758835918762094.

5. Yang SR, Schultheis AM, Yu H, Mandelker D, Ladanyi M, Büttner R. Precision medicine in non-small cell lung cancer: Current applications and future directions. Semin Cancer Biol. 2020. https://doi.org/10.1016/j.semcancer.2020.07.009.

6. Kang YT, Niu Z, Hadlock T, Purcell E, Lo TW, Zeinali M, et al. On-Chip Biogenesis of Circulating NK Cell-Derived Exosomes in Non-Small Cell Lung Cancer Exhibits Antitumoral Activity. Adv Sci (Weinh). 2021;8(6):2003747. https://doi.org/10.1002/advs.202003747.

7. Sanmamed MF, Nie X, Desai SS, Villaroel-Espindola F, Badri T, Zhao D, et al. A burned-out CD8+ T-cell subset expands in the tumor microenvironment and curbs cancer immunotherapy. Cancer Discov. 2021. https://doi.org/10.1158/2159-8290.Cd-20-0962.

8. Grégoire C, Chasson L, Luci C, Tomasello E, Geissmann F, Vivier E, et al. The trafficking of natural killer cells. Immunol Rev. 2007;220(1):169-82. https://doi.org/10.1111/j.1600-065X.2007.00563.x.

9. Myers JA, Miller JS. Exploring the NK cell platform for cancer immunotherapy. Nat Rev Clin Oncol. 2021;18(2):85-100. https://doi.org/10.1038/s41571-020-0426-7.

10. Ke W, Zhang L, Dai Y. The role of IL-6 in immunotherapy of non-small cell lung cancer (NSCLC) with immune-related adverse events (irAEs). Thorac Cancer. 2020;11(4):835-39. https://doi.org/10.1111/1759-7714.13341.

11. Toffoli EC, Sheikhi A, Höppner YD, de Kok P, Yazdanpanah-Samani M, Spanholtz J, et al. Natural Killer Cells and Anti-Cancer Therapies: Reciprocal Effects on Immune Function and Therapeutic Response. Cancers (Basel). 2021;13(4):https://doi.org/10.3390/cancers13040711.

12. Liu L, Bai H, Wang C, Seery S, Wang Z, Duan J, et al. Efficacy and Safety of First-Line Immunotherapy Combinations for Advanced Non-Small-Cell Lung Cancer: A Systematic Review and Network MetaAnalysis. J Thorac Oncol. 2021. https://doi.org/10.1016/j.jtho.2021.03.016.

13. Tarazona R, Sanchez-Correa B, Casas-Avilés I, Campos C, Pera A, Morgado S, et al. Immunosenescence: limitations of natural killer cell-based cancer immunotherapy. Cancer Immunol Immunother. 2017;66(2):233-45. https://doi.org/10.1007/s00262-016-1882-x.

14. Niu C, Li M, Zhu S, Chen Y, Zhou L, Xu D, et al. PD-1-positive Natural Killer Cells have a weaker antitumor function than that of PD-1-negative Natural Killer Cells in Lung Cancer. Int J Med Sci. 2020;17(13):1964-73. https://doi.org/10.7150/ijms.47701.

15. Yamamoto Y, Miyazato K, Takahashi K, Yoshimura N, Tahara H, Hayakawa Y. Lung-resident natural killer cells control pulmonary tumor growth in mice. Cancer Sci. 2018;109(9):2670-76. 
https://doi.org/10.1111/cas.13703.

16. Luna JI, Grossenbacher SK, Murphy WJ, Canter RJ. Targeting Cancer Stem Cells with Natural Killer Cell Immunotherapy. Expert Opin Biol Ther. 2017;17(3):313-24.

https://doi.org/10.1080/14712598.2017.1271874.

17. Xing S, Ferrari de Andrade L. NKG2D and MICA/B shedding: a 'tag game' between NK cells and malignant cells. Clin Transl Immunology. 2020;9(12):e1230. https://doi.org/10.1002/cti2.1230.

18. Key PN, Germino J, Yang L, Piersma SJ, Tripathy SK. Chronic Ly49H Receptor Engagement in vivo Decreases NK Cell Response to Stimulation Through ITAM-Dependent and Independent Pathways Both in vitro and in vivo. Front Immunol. 2019;10(1692 https://doi.org/10.3389/fimmu.2019.01692.

19. Nayyar G, Chu Y, Cairo MS. Overcoming Resistance to Natural Killer Cell Based Immunotherapies for Solid Tumors. Front Oncol. 2019;9(51 https://doi.org/10.3389/fonc.2019.00051.

20. Niogret C, Miah SMS, Rota G, Fonta NP, Wang H, Held W, et al. Shp-2 is critical for ERK and metabolic engagement downstream of IL-15 receptor in NK cells. Nat Commun. 2019;10(1):1444. https://doi.org/10.1038/s41467-019-09431-3.

21. Sun H, Huang Q, Huang M, Wen H, Lin R, Zheng M, et al. Human CD96 Correlates to Natural Killer Cell Exhaustion and Predicts the Prognosis of Human Hepatocellular Carcinoma. Hepatology. 2019;70(1):168-83. https://doi.org/10.1002/hep.30347.

22. Chan CJ, Martinet L, Gilfillan S, Souza-Fonseca-Guimaraes F, Chow MT, Town L, et al. The receptors CD96 and CD226 oppose each other in the regulation of natural killer cell functions. Nat Immunol. 2014;15(5):431-8. https://doi.org/10.1038/ni.2850.

23. Guillerey C, Huntington ND, Smyth MJ. Targeting natural killer cells in cancer immunotherapy. Nat Immunol. 2016;17(9):1025-36. https://doi.org/10.1038/ni.3518.

24. Chauvin JM, Zarour HM. TIGIT in cancer immunotherapy. J Immunother Cancer. 2020;8(2):https://doi.org/10.1136/jitc-2020-000957.

25. Li M, Xia P, Du Y, Liu S, Huang G, Chen J, et al. T-cell immunoglobulin and ITIM domain (TIGIT) receptor/poliovirus receptor (PVR) ligand engagement suppresses interferon-ү production of natural killer cells via $\beta$-arrestin 2-mediated negative signaling. J Biol Chem. 2014;289(25):17647-57. https://doi.org/10.1074/jbc.M114.572420.

26. Romero D. Immunotherapy. PD-1 says goodbye, TIM-3 says hello. Nat Rev Clin Oncol. 2016;13(4):202-3. https://doi.org/10.1038/nrclinonc.2016.40.

27. Wang H, Yan Z, Hao J, Yang B, Wang J, Yi L, et al. CD137 ligand feedback upregulates PD-L1 expression on lung cancer via T cell production of IFN-y. Thorac Cancer. 2019;10(12):2225-35. https://doi.org/10.1111/1759-7714.13207.

28. Lepletier A, Madore J, O'Donnell JS, Johnston RL, Li XY, McDonald E, et al. Tumor CD155 Expression Is Associated with Resistance to Anti-PD1 Immunotherapy in Metastatic Melanoma. Clin Cancer Res. 2020;26(14):3671-81. https://doi.org/10.1158/1078-0432.Ccr-19-3925.

29. Jin S, Deng Y, Hao JW, Li Y, Liu B, Yu Y, et al. NK cell phenotypic modulation in lung cancer environment. PLoS One. 2014;9(10):e109976. https://doi.org/10.1371/journal.pone.0109976. 
30. Aktaş ON, Öztürk AB, Erman B, Erus S, Tanju S, Dilege Ş. Role of natural killer cells in lung cancer. J Cancer Res Clin Oncol. 2018;144(6):997-1003. https://doi.org/10.1007/s00432-018-2635-3.

31. Tao L, Wang S, Yang L, Jiang L, Li J, Wang X. Reduced Siglec-7 expression on NK cells predicts NK cell dysfunction in primary hepatocellular carcinoma. Clin Exp Immunol. 2020;201(2):161-70. https://doi.org/10.1111/cei.13444.

\section{Tables}

Table 1 Clinical and demographic characteristics of the study populations

\begin{tabular}{|c|c|c|}
\hline Characteristic & $\begin{array}{l}\text { No. }(\%) \text { of healthy participants } \\
(n=67)\end{array}$ & $\begin{array}{l}\text { No. }(\%) \text { of patients with NSCLO } \\
(n=56)\end{array}$ \\
\hline \multicolumn{3}{|l|}{ Gender } \\
\hline Female & $27(40.3)$ & $21(37.5)$ \\
\hline Male & $40(59.7)$ & $35(62.5)$ \\
\hline \multicolumn{3}{|l|}{ Age } \\
\hline$<65$ years & $29(43.3)$ & $23(41.1)$ \\
\hline$\geq 65$ years & $38(56.7)$ & $33(59.0)$ \\
\hline \multicolumn{3}{|c|}{ Smoking status } \\
\hline Ever-smoker & $19(28.4)$ & $29(51.8)$ \\
\hline Never-smoker & $48(71.6)$ & $27(48.2)$ \\
\hline \multicolumn{3}{|l|}{ Stage } \\
\hline ૫ & & $8(14.3)$ \\
\hline ૧ & & $21(37.5)$ \\
\hline ૧ & & $13(23.2)$ \\
\hline ૫ & & $14(25.0)$ \\
\hline \multicolumn{3}{|c|}{$\begin{array}{l}\text { ECOG performance } \\
\text { status }\end{array}$} \\
\hline $0-1$ & & $47(84.0)$ \\
\hline $2-4$ & & $9(16.1)$ \\
\hline \multicolumn{3}{|c|}{ Histologic subtype } \\
\hline$A D C$ & & $27(48.2)$ \\
\hline SQCC & & $29(51.8)$ \\
\hline
\end{tabular}


Abbreviations: ADC, adenocarcinoma; ECOG, Eastern Cooperative Oncology Group; NSCLC, non-small cell lung cancer; SQCC, squamous cell carcinoma

Table 2 Association between NK cell surface receptors and disease progression, histologic types of nonsmall cell lung cancer

\begin{tabular}{|c|c|c|c|c|c|c|}
\hline \multirow[t]{2}{*}{ NK cell subset } & \multicolumn{3}{|c|}{ Cancer stage } & \multicolumn{3}{|c|}{ Histologic type } \\
\hline & Early (\%) & Late (\%) & P-value & ADC (\%) & SQCC (\%) & P-value \\
\hline IFN- $\gamma^{+}$ & $28.74 \pm 6.37$ & $6.07 \pm 2.14$ & 0.010 & $14.74 \pm 6.94$ & $19.18 \pm 7.22$ & 0.685 \\
\hline TIGIT & $49.27 \pm 5.89$ & $66.41 \pm 4.50$ & 0.039 & $55.94 \pm 5.58$ & $57.57 \pm 6.58$ & 0.851 \\
\hline TIM-3 $^{+}$ & $28.65 \pm 3.98$ & $61.08 \pm 5.93$ & 0.004 & $41.97 \pm 7.21$ & $57.33 \pm 11.70$ & 0.268 \\
\hline PD- $1^{+}$ & $1.58 \pm 0.27$ & $4.53 \pm 1.11$ & 0.009 & $2.79 \pm 0.78$ & $2.94 \pm 0.91$ & 0.899 \\
\hline $\mathrm{CD} 6^{+}$ & $64.70 \pm 1.98$ & $89.07 \pm 3.83$ & 0.005 & $74.95 \pm 7.43$ & $80.75 \pm 12.15$ & 0.687 \\
\hline NKP30 ${ }^{+}$ & $34.08 \pm 4.08$ & $28.03 \pm 4.06$ & 0.310 & $38.46 \pm 4.13$ & $24.38 \pm 3.09$ & 0.011 \\
\hline
\end{tabular}

Abbreviations: ADC, adenocarcinoma; NK, natural killer; SQCC, squamous cell carcinoma.

\section{Figures}


A
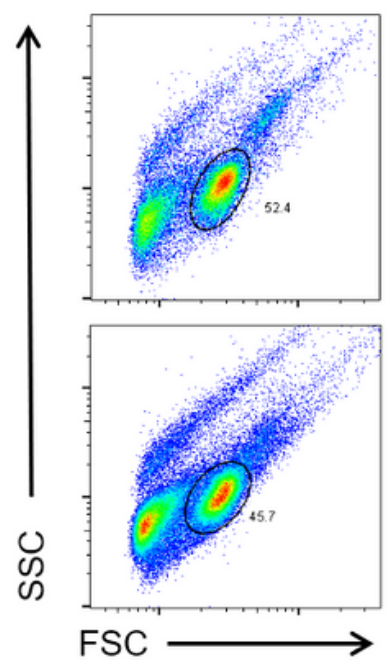

B

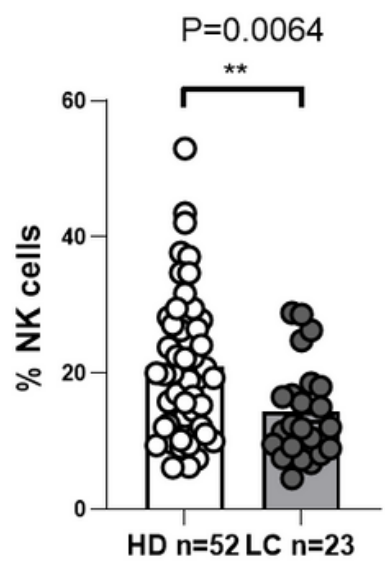

E

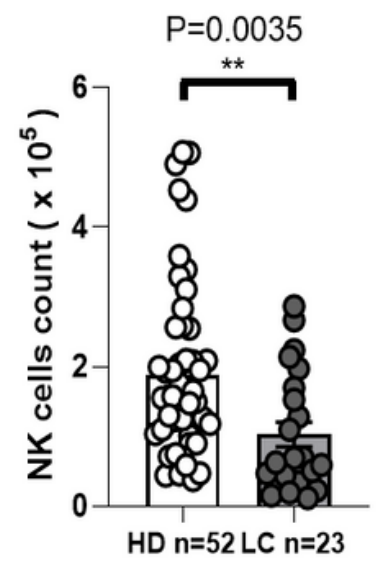

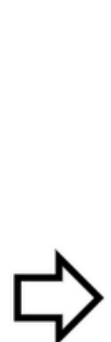

อี
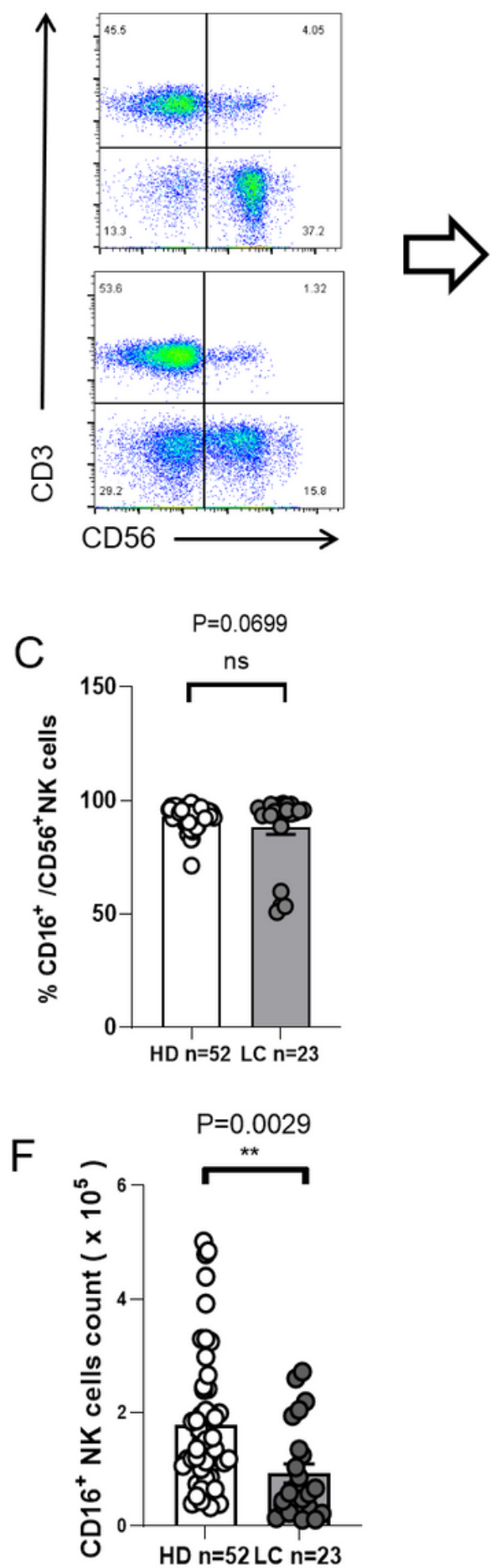

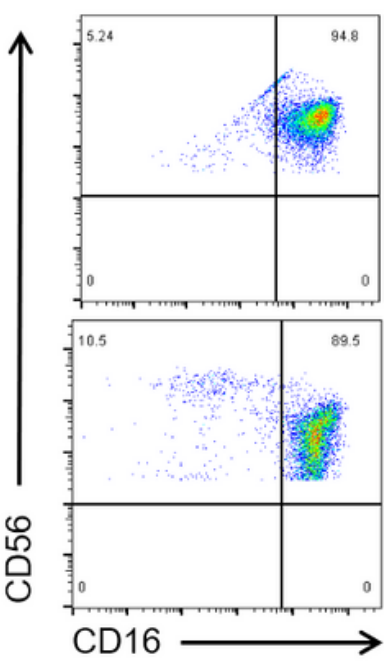

HD

LC
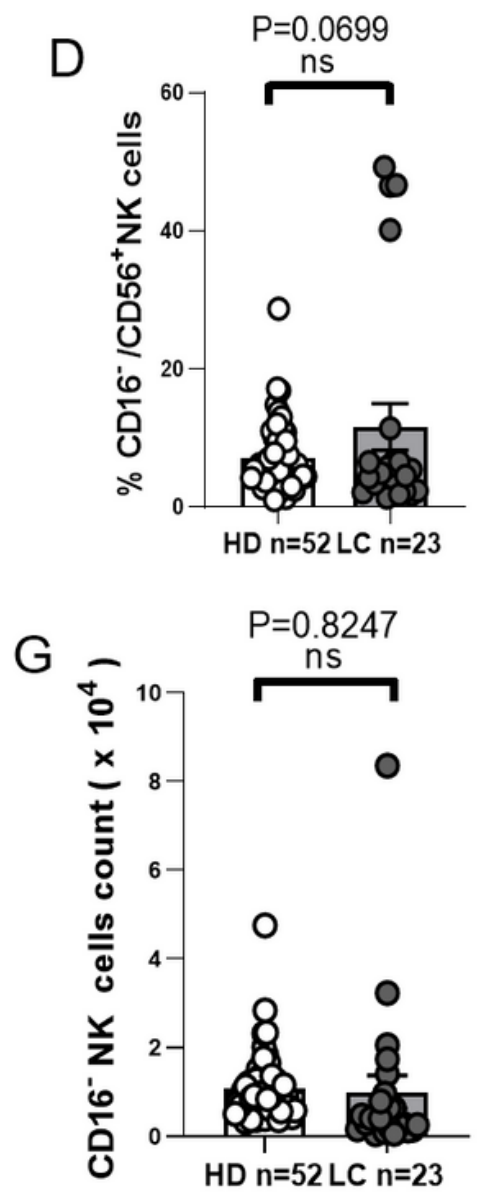

Figure 1

Total number of NK cells in peripheral blood decreases in NSCLC patients. Flow cytometric gating strategy used to analyze NK cell subsets in PBMC samples (A). LC represents lung cancer and HD, healthy donors. Percentage of NK cells in the lymphocyte population (B) and the ratios of $\mathrm{CD}^{+} 6^{+}(\mathrm{C})$ and $\mathrm{CD}^{-} 6^{-}$ (D) to CD $56^{+}$NK cells in NSCLC patients vs. healthy participants. Numbers of NK cell (E), CD $16^{+} \mathrm{NK}$ cell $(\mathrm{F})$, and $\mathrm{CD}_{16^{-}} \mathrm{NK}$ cell (G) in NSCLC patients and healthy participants peripheral blood. 


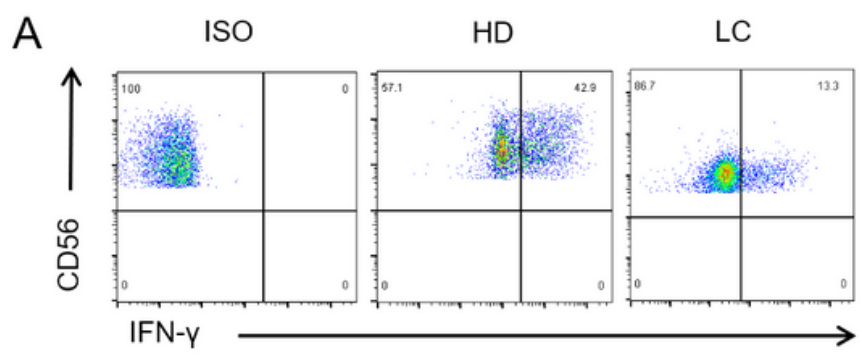

C
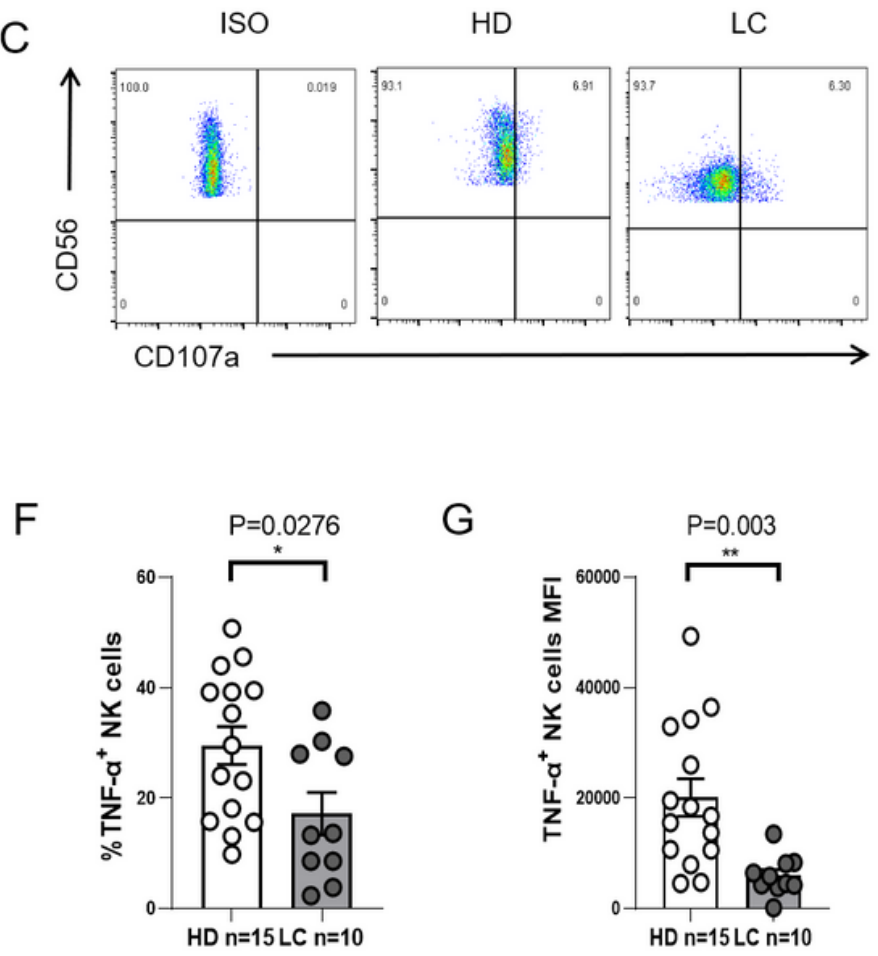

\begin{abstract}
G
\end{abstract}

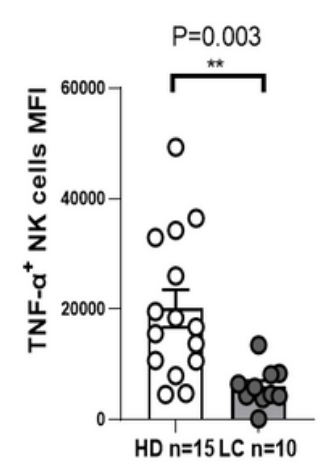

B

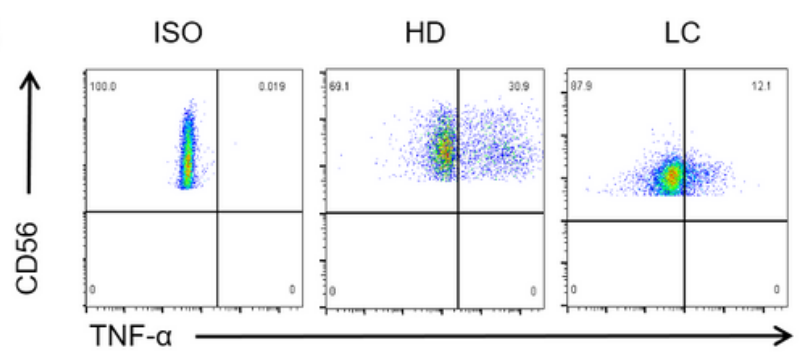

D
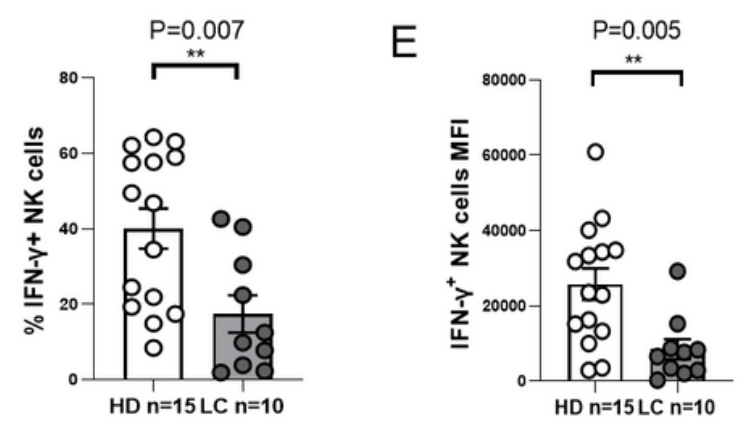

$\mathrm{H}$

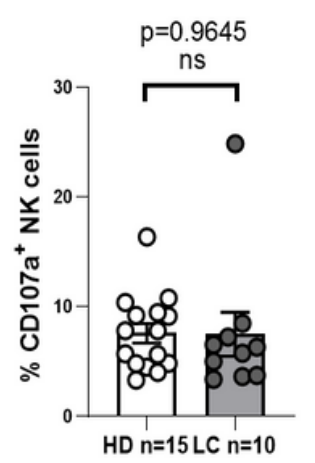

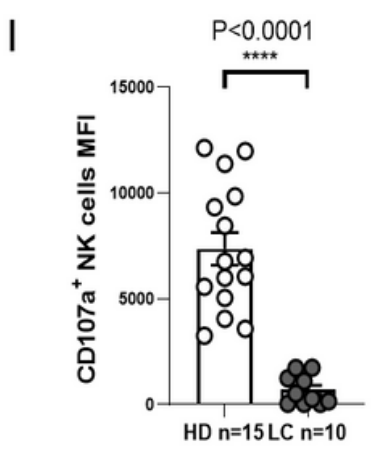

Figure 2

\section{NK cell function decreases in NSCLC patients.}

Representative flow cytometry density plots for IFN-y (A), TNF-a (B), and CD107a (C) expression on NK cells in NSCLC patients and control participants. LC represents lung cancer; HD, healthy donors; and ISO, isotype. The proportion of IFN ${ }^{+}(D)$, TNF- $^{+}(\mathrm{F})$, and CD107a ${ }^{+}(\mathrm{H}) \mathrm{NK}$ cells in NSCLC patients and healthy participants. Median fluorescence intensity (MFI) of IFN ${ }^{+}(\mathrm{E}), \mathrm{TNF}^{+} \mathrm{a}^{+}(\mathrm{G})$, and CD107a ${ }^{+}(\mathrm{I}) \mathrm{NK}$ cells. 
A

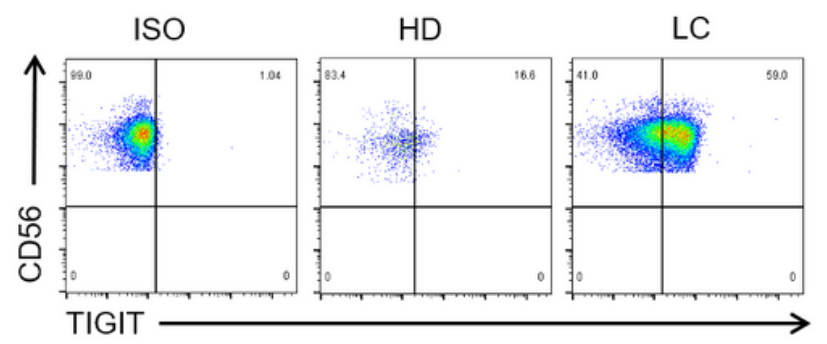

C

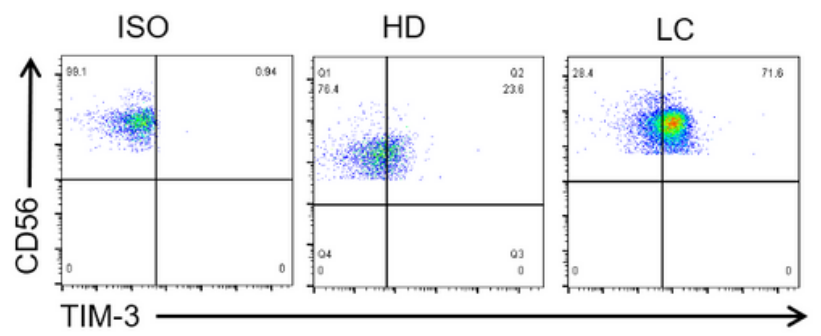

E

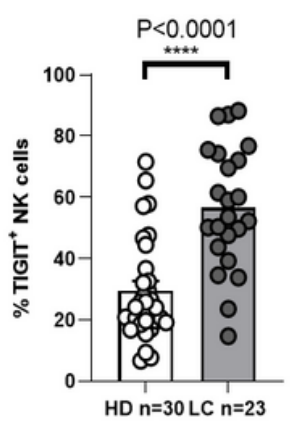

I

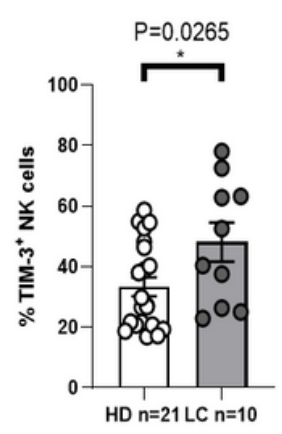

F

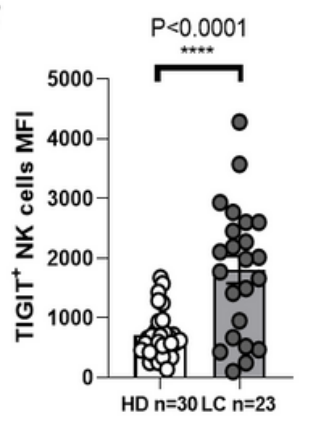

J

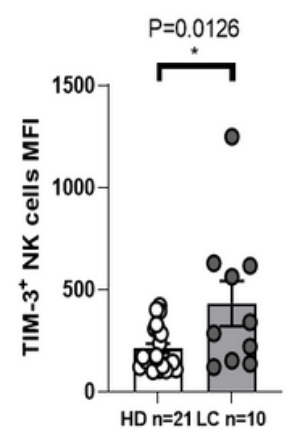

B

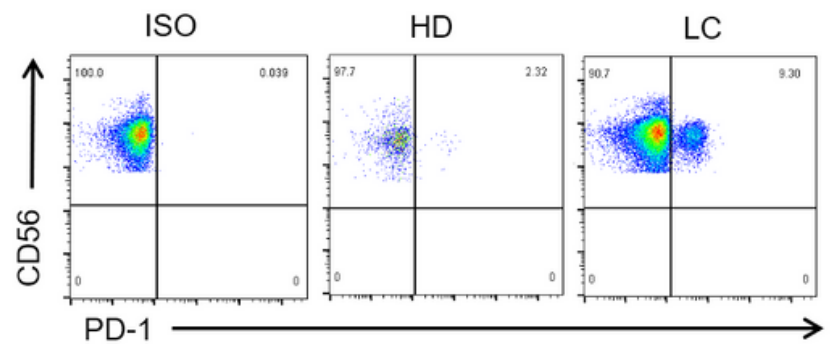

D

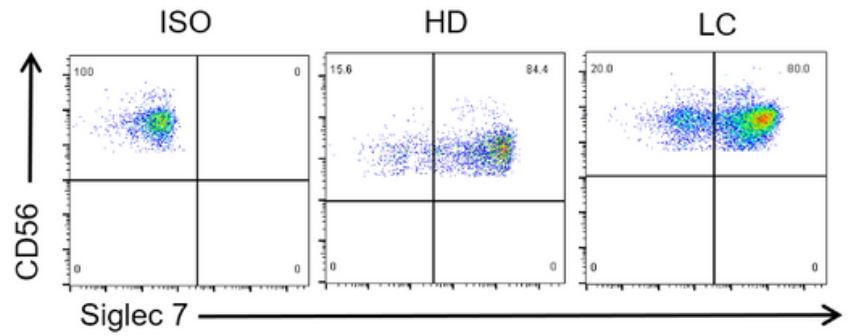

G

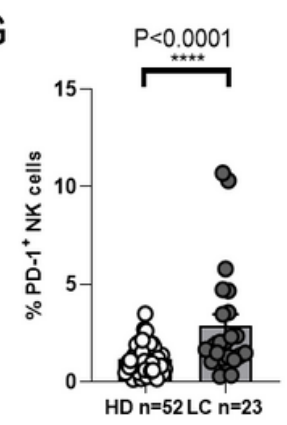

$\mathrm{H}$

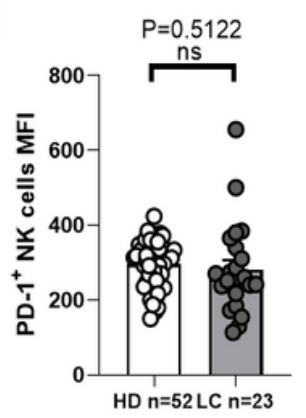

K

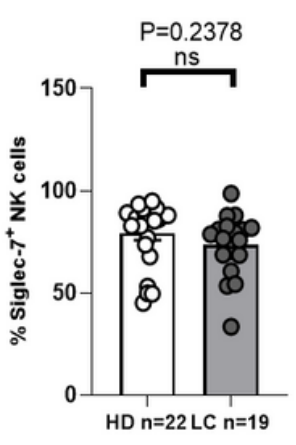

L

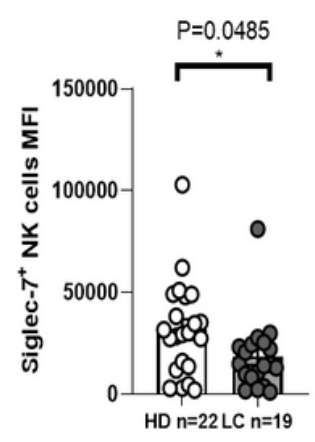

Figure 3

Abnormal expression of inhibitory receptors on the surface of NK cells in NSCLC patients.

Representative flow cytometry density plots for TIGIT (A), PD-1 (B), TIM-3 (C), and Siglec-7 (D) expression on NK cells from NSCLC patients and healthy participants. LC represents lung cancer; HD, healthy donors; and ISO, isotype. Proportion of $\mathrm{TIGIT}^{+}(\mathrm{E}), \mathrm{PD}^{-1^{+}}(\mathrm{G}), \mathrm{TIM}^{+}{ }^{+}(\mathrm{I})$ and Siglec-7 ${ }^{+}(\mathrm{K})$ NK cells in patients with NSCLC and healthy participants. Median fluorescence intensity (MFI) of $\mathrm{TIGIT}^{+}(\mathrm{F}), \mathrm{PD}-1^{+}(\mathrm{H}), \mathrm{TIM}-3^{+}(\mathrm{J})$, and Siglec- $7^{+}(L)$ NK cells in patients with NSCLC and healthy participants. 


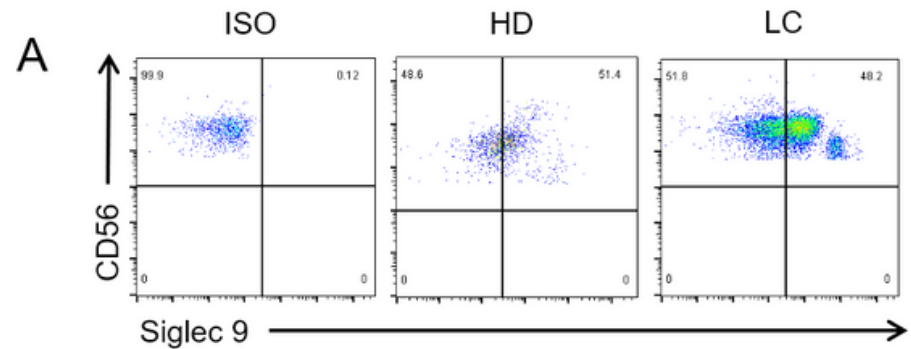

C

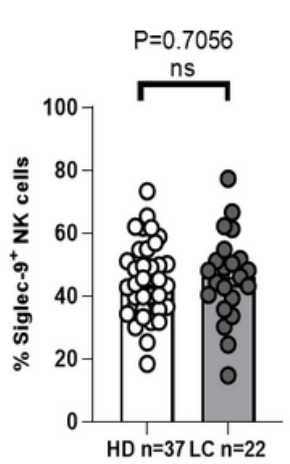

D

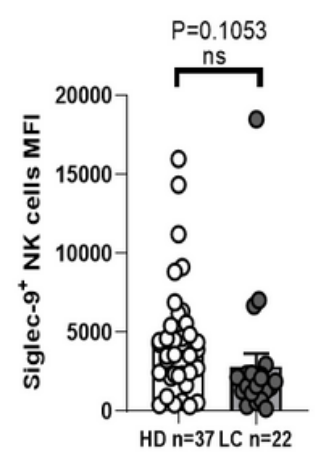

B

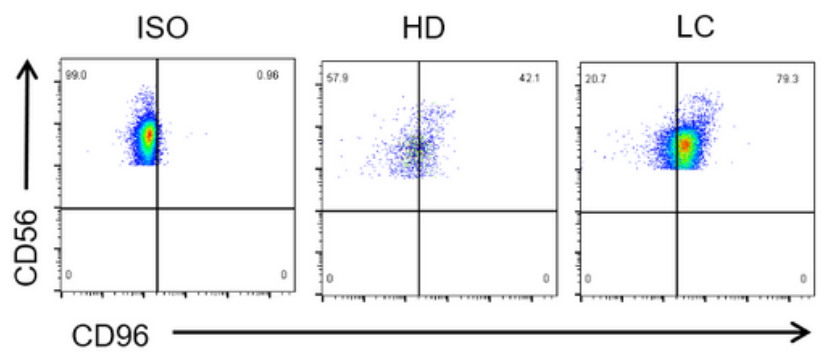

$\mathrm{E}$

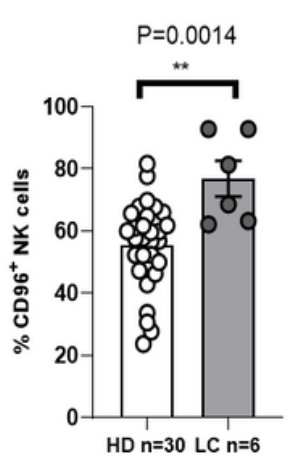

$\mathrm{F}$

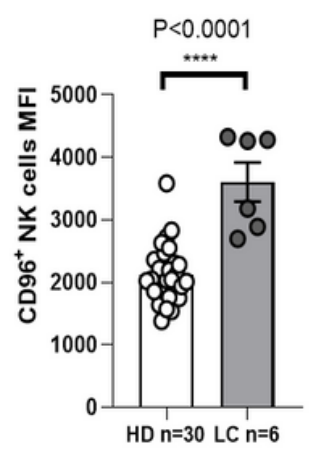

Figure 4

Abnormal expression of CD96 but not Siglec-9 inhibitory receptors on the surface of NK cells in NSCLC patients.

Representative flow cytometry density plots for Siglec-9 (A) and $\mathrm{CD}^{+} 6^{+}(\mathrm{B})$ expression on NK cells in NSCLC patients and control participants. LC represents lung cancer; HD, healthy donors; and ISO, isotype. Proportion of Siglec- $9^{+}(C)$ and CD $96^{+}$(E) NK cells in patients with NSCLC and healthy participants. Median fluorescence intensity of Siglec-9 $9^{+}(\mathrm{D})$ and CD $96^{+}(\mathrm{F})$ NK cells in patients with NSCLC and healthy participants. 
A

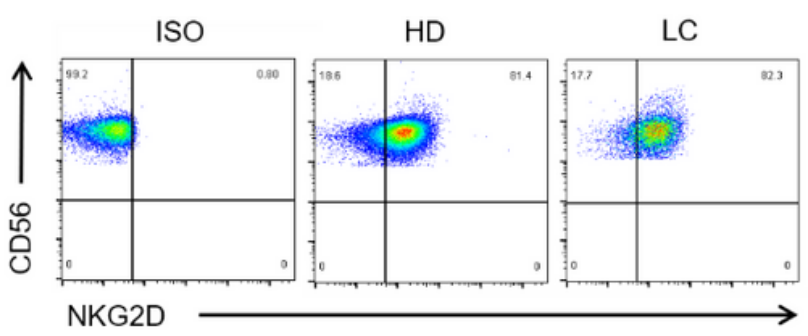

C

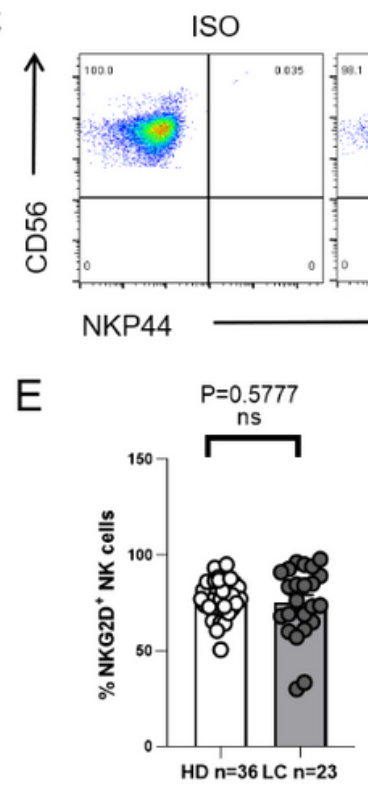

I

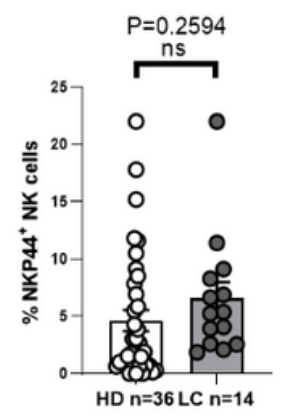

HD LC
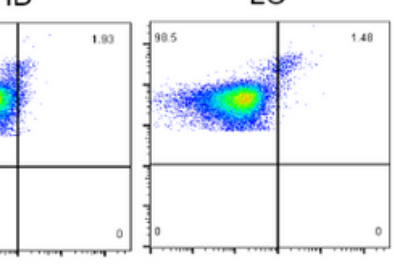

F

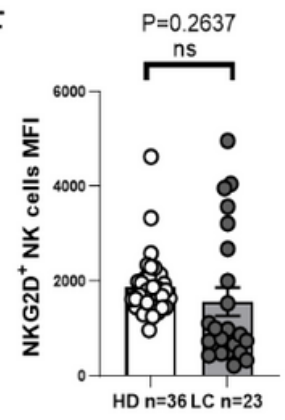

J

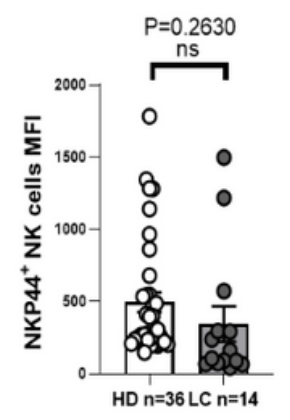

B

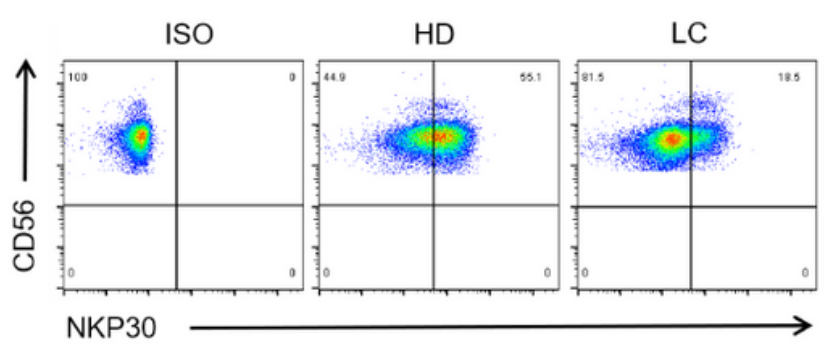

D

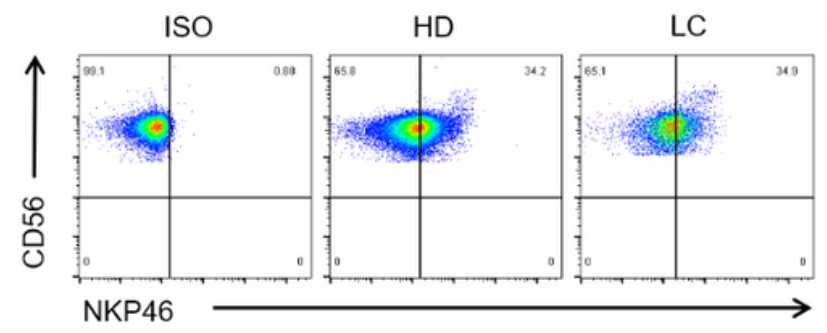

G

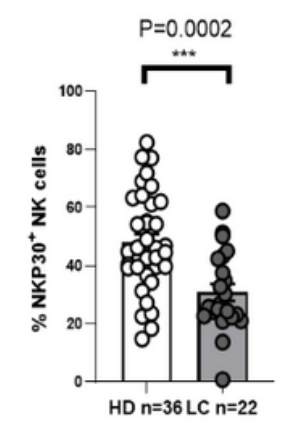

$\mathrm{H}$

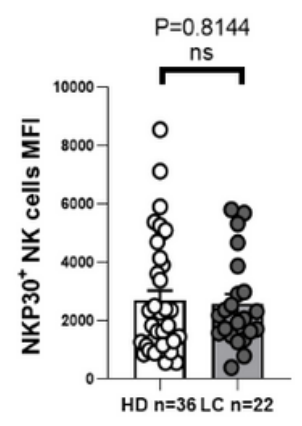

K
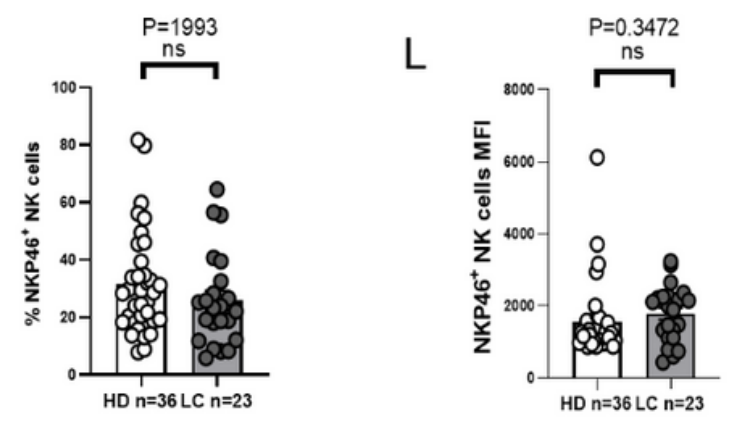

\section{Figure 5}

\section{Abnormal expression of activating receptors on the surface of NK cells in NSCLC patients.}

Representative flow cytometry density plots of $\mathrm{NKG}_{2} \mathrm{D}^{+}(\mathrm{A}), \mathrm{NKP}^{+} \mathrm{O}^{+}(\mathrm{B}), \mathrm{NKP}_{4} 4^{+}(\mathrm{C})$, and $\mathrm{NKP}_{4}{ }^{+}$(D) NK cells in NSCLC patients and healthy participants. LC represents Lung cancer; $H D, X X X$; and ISO, Isotype

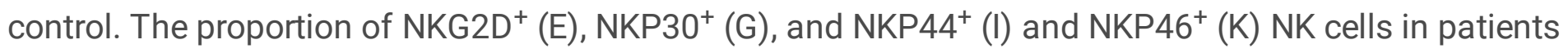

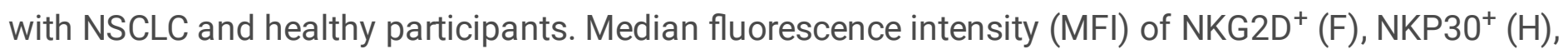
$\mathrm{NKP}_{4} 4^{+}(\mathrm{J})$, and NKP46${ }^{+}(\mathrm{L})$ NK cells in patients with NSCLC and healthy participants. 